\title{
Study of Multiband DRA for Mobile communication
}

\author{
Dorik Narayan Yadav \\ Department of Physics, Prithivi Narayan Campus, Pokhara, Nepal
}

\begin{abstract}
The explosive demand for mobile communication and information transfer using personal devices such as mobile phone or notebook computer has caused the need for major advancements of antenna design. With the development of $3 G$ and $4 G$ technologies multiband and wideband antennas operating at additional frequency band such as UMTS and LTE are required. In this chapter it is initially presented the fundamental parameters of antenna to be taken into account while designing an antenna and determining the operating frequency bands. Multiband antennas which are used especially in mobile unit are described. The techniques to make an antenna convenient for multiband operations are given. There different antennas such as monopoles, PIFA are examined with several examples in the literatures. In the last part, the types of wideband antennas (microsstrip patch antenna, DRA of planer) used in mobile communication which are more appropriate for base station or access point applications, are presented.
\end{abstract}

Keywords: UMTS and LTE, PIFAs, DRA and 4G technologies.

\section{INTRODUCTION:}

The popularity of mobile communication system has increased during the last decate and market demand continues to increase. Antenna is one of the most important fundamental part of there systems. An antenna is a device, which transforms the electromagnetic waves in antenna to radiating waves in an unbounded medium such as air in transmitting mode and vice versa in receiving mode. Since antennas depend of frequency so designed to operate for certian frequency bands. Earlier, mobile systems were designed to operate for one of the frequency bands of $2 \mathrm{G}$ (second generation) system which are Digital Cellular System (DCS) Personal Communication Service (PCS) and Global System for Mobile Communication (GSM) networks. But currently because of rapid growth of mobile communication systems has forced to use of novel antennas for base and mobile station applications. Thus many mobile communications systems use several frequency bands such as GSM 900/1800/1900 bands (890-960MHZ and 17101990 MHZ) Universal Mobile Telecommunication Systems (UMTS) and UMTS 3G expansion bands (1900-2200 MHZ and 2500-2700MHZ), and Wi-Fi (Wireless Fidelity) / Wireless Local Area Networks (WLAN) bands (2400-2500MHZ and $5100-5800 \mathrm{MHZ}$ ) where the list of frequently used frequency bands is given in literature (Best 2008).
Conventionally, all the frequency bands of mobile communication can not be operated by single antenna thus multiple different antennas covering these bands separately should be used. However uses of many antennas is usually limited by the volume and cost constraints of the applications. Thus multiband and wideband antennas are necessary to provide multifunctional operations for mobile communication. In a mobile communication system a multiband antenna can be defined as the antenna operation at distinct frequency bands, but not at the intermediate frequencies between bands. For example, a triple band antenna for GSM $900 / 1800 / 1900$ bands can cover the frequency bands 890-960 MHZ and 1710-1990 MHZ however, it does not operate properly at the frequency such as 1200 MHZ or 2500 MHZ. Where a wideband antenna operates at every frequency points within a given frequency band. For example a wideband antenna covering UMTS extended UMTS and WLAN 2400 bands functions at every frequency points.

\section{CROSS POLAR DISCRIMINATION:}

It is demanded that each antenna port receives signals only form its designated linear polarization (co-polarization) however in practical antennas also receive unwanted signals from orthogonal polarization called as cross polarization. The ratio of received $\mathrm{Ln}_{\mathrm{n}}$-polar signal level to cross polar signal lev $d x$ is called cross polar discrimination. For constant cross polarization discrimination within 
beamwidth is difficult that this value falls to $20 \mathrm{~dB}$ for $60^{\circ}$ degress in principal $\mathrm{H}$-plane. The required cross polar discrimination for mobile communication system is around $25 \mathrm{~dB}$ in boresight direction and $10 \mathrm{~dB}$ at the edges of beamwidth.

\section{INTERMODULATION:}

When the signals with multiple frequencies $\left(f_{1}\right.$, $\left.f_{2}, f_{3}, \ldots \ldots f_{n}\right)$ are received by a nonlinear device, intermodulation frequency terms $\left(f_{1}-f_{2}, f_{1}+f_{2} 2 f_{1}\right.$ $\left.f_{2}, \ldots . . ..\right)$ are generated. An antenna is a linear device but it may slightly deviate from linearity when high power is transmitted or received by the antenna. This nonlinearity is formed due to mechanical joints or nonliner materials used in the antenna. The intermodulation level is critical in base station applications since the intermodulation frequencies can degrade the quality of communication system. Intermodulation frequency terms fall in the frequency band of interest. For example two transmitted frequencies $\left(f_{1=} 935 \mathrm{MHZ}\right.$ and $f_{2}=955$ MHZ) in frequency band of GSM 900 can generate $3^{\text {rd }}$ order intermodulation term at the frequency $2 \mathrm{f}_{1}-\mathrm{f}_{2}=915 \mathrm{MHZ}$ which again falls into GSM 900 band. Thus the intermodulation levels are low as far as possible that the signal level for base station application is between $-180 \mathrm{dBc}$ and $-120 \mathrm{dBc}$. (dBc: Power in $\mathrm{dB}$ scale relative to carrier power) Incase of application of mobile phone or notebook computer the intermodulation terms are usually ignored.

\section{Specific Absorption rate (SAR):}

Some portion of transmitted power is absorbed by human body since antenna of mobile phone and notebook computer is nearby human body. The specific absorption rate (SAR) is defined as the absorbed power density at the particular point of the human body. SAR can be quantitatively expressed as where $\mathrm{dP}_{\text {abs }}$ is absorbed power within an infinitesimal volume of $\mathrm{dV} ; \mathrm{E}$ is the peak electric field strength within $\mathrm{dV} ; \rho$ and $\sigma$ are mass density and conductivity of the human body SAR is important that certain regulations about SAR, Which are based on the biological effects of thermal heating due to radiation should be satisfied. The IEEE standard aboutSAR indicates the maximumallowed 1-g averaged maximum SAR is $1.6 \mathrm{~W} / \mathrm{kg}$ and whole body average peak SAR is $0.8 \mathrm{~W} / \mathrm{kg}$. 10 -g averaged maximum SAR value is commonly used as $2 \mathrm{~W} / \mathrm{kg}$ in Europe countries.

\section{Multiband Antennas for Mobile Communication:}

A wide variety of antenna type uses different multiband techniques for multiband operation.

\section{MULTIBAND TECHNIQUES \\ Higher order Resonances:}

One of the basic ways of getting multiband operation is to utilize from higher order resonances. A monopole antenna is often used with length of $\lambda / 4$. For this case the antenna resonates at $f_{0}$ with electric field minimum at the feed. However a similar condition of minimum electric field at the feed also exists when same antennas length corresponds to $3 \lambda / 4$. Hence the monopole antenna also resonates at $3 \mathrm{f}_{0}$ other higher resonances also exist at higher frequencies such as $5 \mathrm{f}_{0}$. Higher order resonances are used in many types of antennas such as dipoles, helices, patches and slots. Multiband and wideband antennas for mobile communication systems mounted on a typical mobile phone is given. According to the results, the antenna has the resonances at frequencies $f_{0}$ and $2.6 f_{0}$ that higher order resonances principle almost holds for this case.

\section{Multiple Resonant Structures:}

The most popular technique for obtaining multiband antenna system is the usage of multiple resonant structures. Here, two or more resonant structures, which are closely located in space or even co-located with the single feed are used. They are typical examples for corporate feed that two resonate structures are excited simultaneously. Also multiple resonate structures that the second resonate structure can be excited after the first structure are excited. The multiple resonate structure technique is frequently used in mobile communication system to achieve multiband mobile antennas. For example dual frequency antenna systems for handsets are proposed. The designed structures are the combination of monopole and helical antennas that multiple resonances at two different frequencies are acquired for dual-band operation at GSM 900 and 1800 bands. 


\section{CONCLUSION:}

In conclusion the engineers interested in mobile communication acquire an initial comprehension about fundamentals and characteristics of multiband wideband antennas used in mobile communication systems. The readers can utilize from the given references for more detail.

\section{REFERENCES:}

1. Ali, M., oKoniewski, M., stuchly, M.A. And Stuchly, M.

M 1999 Dual - frequency strip - sleeve monopole for laptop computer, IEEE transactions on antennas and propagation 47(2): 317-323
2. Balanis, C.A. 2005. Antenna theory; analysis and design John Wiley \& Sons. Inc; New York, USA.

3. Chen, H.D. and Chen, H.T. 2004 Acpw-fed dualfrequency monopole antenna. IEEE transactions on antennas and propagation, 52(4):978-982.

4. Guterman J. Moreira, A.A. and Peixerio, C. 2006 IEEE antennas and wireless propagation letters, 5(1): 141-144.

5. Katsibas, K.D. Balanis, C.A. Tirkas P.A. and Birtcher, C.R. 1998

Folded loop antenna for mobile hand held units, IEEE transactions on antennas and propagation, 46(2): 260-266. 